\title{
Left atrial and left atrial appendage remodeling after transcatheter aortic valve replacement: Preliminary results
}

\author{
Tian-Yuan Xiong ${ }^{1,2}$, Fei Chen ${ }^{1}$, Yi-Jian $\mathrm{Li}^{1}$, Yuan Feng ${ }^{1}$, Mao Chen ${ }^{1}$ \\ ${ }^{1}$ Department of Cardiology, West China Hospital, Sichuan University, Chengdu, PR China \\ ${ }^{2}$ State Key Lab of Hydraulics and Mountain River Engineering, Sichuan University, Chengdu, PR China
}

Atrial fibrillation (AF) is a common comorbidity in transcatheter aortic valve replacement (TAVR) recipients. The reported incidence of preexisting $\mathrm{AF}$ in this patient population ranges from $34 \%$ to $49 \%$, while new-onset AF after TAVR is also not rare (6.8-8.6\%). Both carries with an increased risk of mortality and stroke [1]. It is recognized that the assessment of the left atrial (LA) and left atrial appendage (LAA) anatomy and function has important prognostic implications in $\mathrm{AF}$ and the risk of stroke [2]. The relief from pressure overload by TAVR might help in reverse remodeling of LA and LAA, but no study to date has evaluated the sequential change of these structures post-TAVR. Such information should be of interest as bias exists when diagnosing new-onset $\mathrm{AF}$ by ambulatory electrocardiogram or the ICD-9 code during any re-hospitalization. Thus, the present study sought to report our preliminary results on the 1-year volume changes of LA and LAA post-TAVR.

The volume of LA and LAA were retrospectively assessed, LAA morphology and take-off position in 43 consecutive TAVR recipients due to symptomatic severe aortic stenosis from multislice computed tomography (MSCT) performed pre-procedurally (referred to as pre-TAVR), post-procedurally before discharge (referred to as inhospital) and 1-year post-TAVR. This study was approved by the institutional review board. Written informed consent were obtained from all patients.

Acquisition and reconstruction of MSCT scans in the documented center have been described previously [3]. Mimics 21.0 (Materialise NV, Leuven, Belgium) was used to perform three-dimensional reconstruction, segmentation and volume cal- culation of LA and LAA in systole as previously described [4]. LAA morphology was classified into chicken-wing (CW) and non-CW type [5]. LAA positions were classified based on the superior aspect of the LAA orifice with that of the left superior pulmonary vein orifice as high, middle, or low [5]. Reverse remodeling was defined as an absolute change in volume, while discordant remodeling was defined as an increase in LAA volume but a decrease in LA volume or vice versa. Baseline characteristics and follow-up data were extracted from a dedicated database.

Continuous variables were presented as mean \pm standard deviation or median (interquartile range) as appropriate. Comparisons of volume change at the three points in time were carried by the Friedman M test and followed by post-hoc pairwise multiple comparisons. Intra- and inter-observer reliability in measuring LA and LAA volume were assessed in 10 randomly selected patients from the cohort with intraclass correlation coefficient (ICC). All computations relied on commercially available software (SPSS IBMS v21; SPPS Inc., Chicago, USA), with statistical significance set at two-tailed 0.05.

The mean age of this cohort was $73.9 \pm 6.4$ years. Female patients accounted for $41.9 \%$ of this cohort. Procedural success was achieved in all patients. A total of $7(16.3 \%)$ patients were documented with pre-existing AF. CW-type LAA was identified in $37(86.0 \%)$ patients. There were $4.6 \%, 51.2 \%$ and $44.2 \%$ of patients with a high, mid and low take-off of LAA, respectively. The volume of LA decreased continuously during 1-year follow-up (pre-TAVR vs. in-hospital vs. 1 year: 122.0

Address for correspondence: Prof. Mao Chen, Department of Cardiology, West China Hospital, Sichuan University, \#37 Guoxue Alley, Chengdu, 610041, PR China, tel: 86-28-85423362, fax: 86-28-85423170, e-mail: hmaochen@vip.sina.com 


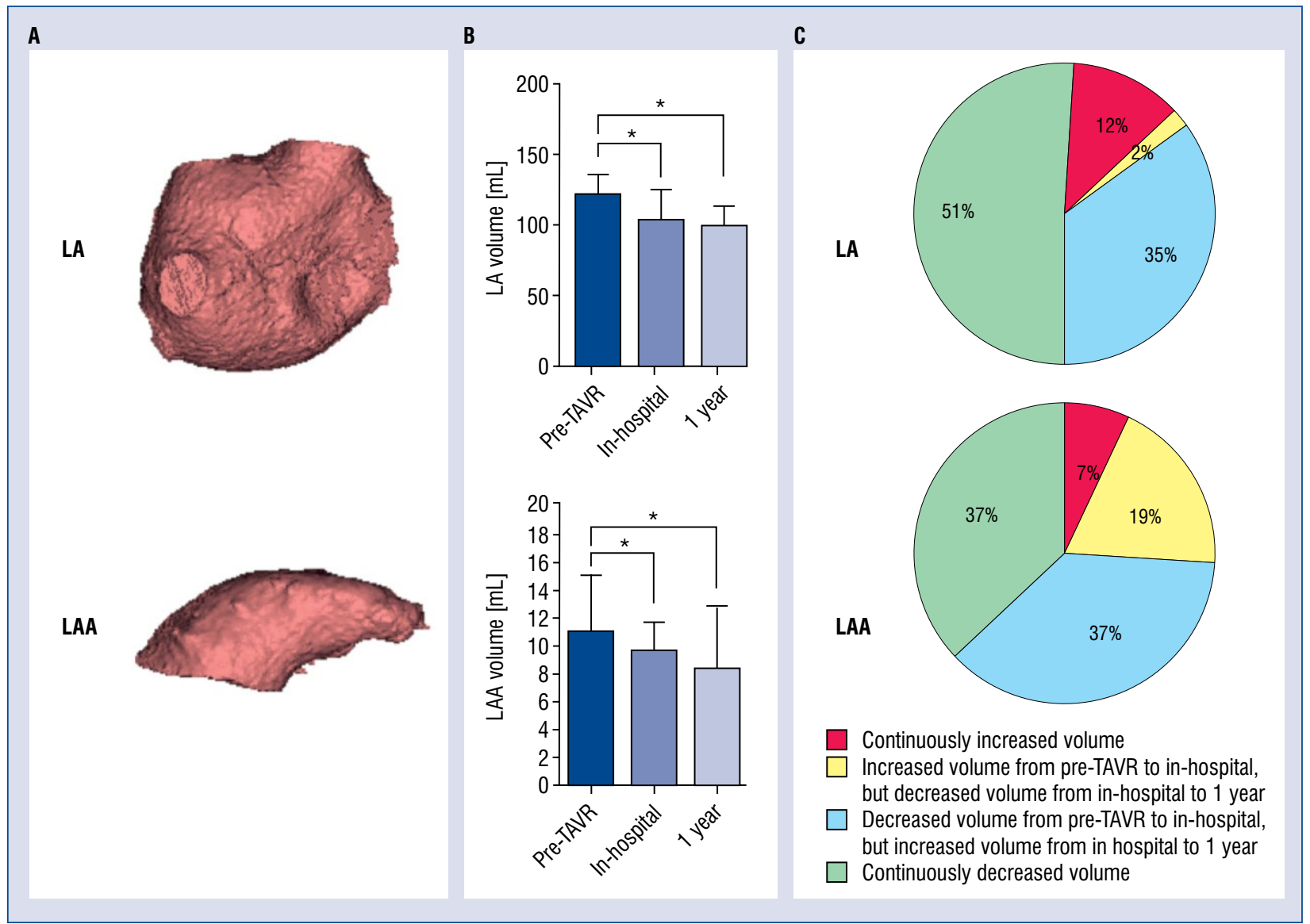

Figure 1. Left atrium (LA) and left atrial appendage (LAA) remodeling pattern post-transcatheter aortic valve replacement (TAVR); A. Three-dimensional segmentation of LA and LAA; B. Serial changes of volumes of LA and LAA during 1-year follow-up post-TAVR; ${ }^{*}$ Stands for reaching statistical significance in post-hoc multiple comparisons; C. The distribution of different patterns of volume change in the LA and LAA post-TAVR.

[44.1] mL vs. 104.5 [43.1] mL vs. 100.7 [33.6] mL, $\mathrm{p}<0.001$ ), so did the volume of LAA (pre-TAVR vs. in-hospital vs. 1 year: 11.0 [6.8] mL vs. 9.7 [4.6] mL vs. 8.4 [6.3] mL, p = 0.001; Fig. 1). In post-hoc multiple comparisons, statistically significant differences in volume was detected between pre-TAVR and in-hospital, but not between in-hospital and 1 year (Fig. 1). On an individual level, the proportion of patients who experienced a continuous decrease in LAA volume was numerically smaller than that for LA volume ( $37.2 \%$ vs. $51.2 \%, \mathrm{p}=0.19$; Fig. 1, illustrating the distribution of 4 different patterns of volume change). A total of 10 (23.3\%) patients showed discordant remodeling between LA and LAA from pre-TAVR to in-hospital, while the number was 17 (39.5\%) from in-hospital to 1 year. At 1 year, a reverse remodeling from preTAVR was achieved in $70 \%$ and $72 \%$ of patients for LA and LAA, respectively.

Intra-observer reliability was excellent for both LAA and LA volume (ICC $0.98,95 \%$ confi- dence interval [CI] 0.93-0.99; ICC 0.99, 95\% CI 0.97-0.99). Inter-observer reliability was good to excellent for LAA volume (ICC 0.94, 95\% CI 0.78-0.99) and excellent for LA volume (ICC 0.99, 95\% CI 0.91-0.99).

The major finding of this study was that TAVR in general brought reverse remodeling of LA and LAA. A more pronounced decrease was seen in a short period before discharge than during the postdischarge 1-year follow-up. This is consistent with a previous study with echocardiography in patients receiving surgical aortic valve replacement (SAVR) for aortic stenosis [6]. However, a volume increase of LA and LAA was observed at either in-hospital from pre-TAVR or 1 year from in-hospital in roughly more than half of the patients, suggesting a dynamic but not constant change of LA and LAA remodeling post-TAVR. Moreover, patients may have discordant volume change between LA and LAA.

Atrial fibrillation is a comorbidity or complication of outcome implications in the TAVR 
population, but the true burden of which is likely underestimated during routine clinical care postprocedurally. In a study involving patients who received permanent pacemaker post-TAVR, a much higher incidence of new-onset $\mathrm{AF}$ was detected with data from device checks and $85 \%$ subclinical new-onset AF was identified 4 weeks beyond TAVR [7]. This finding illustrated current suboptimal surveillance of subclinical $\mathrm{AF}$ and a consequent underuse of anticoagulation therapy, which might translate to a devastating stroke event. Given the structural change of LA and LAA is involved in the onset of $\mathrm{AF}$ and its subsequent stroke risk, a follow-up on LA and LAA with readily available MSCT might provide another perspective to this problem. As demonstrated in our study, not all patients benefited from the relief of pressure overload by TAVR and the process of reverse remodeling was sometimes dynamic and discordant. LA volume index actually increased 1 -year postSAVR in patients with a baseline index $\geq 40 \mathrm{~mL} /$ $/ \mathrm{m}^{2}$ [8]. Around $23 \%$ of SAVR recipients remained with left ventricular hypertrophy and LA dilatation 1 year after the procedure, which was associated with a significantly lower survival rate at 3 years [9]. Structural changes in LA and LAA may also precede the development of AF and thrombus formation. Thus, an early identification of patients who would experience volume increase of LA and LAA might contribute to patient management postTAVR. Further studies to correlate the imaging findings with clinical characteristics and outcomes are needed.

\section{Funding}

This work was supported by West China Hospital "1·3·5" Discipline of Excellence Project - Mechanisms of aortic stenosis and the clinical applications; the fellowship of China Postdoctoral Science Foundation (2020M683327); Open Fund Research from State Key Laboratory of Hydraulics and Mountain River Engineering (SKHL1920).

Conflict of interest: None declared

\section{References}

1. Mentias A, Saad M, Girotra S, et al. Impact of pre-existing and new-onset atrial fibrillation on outcomes after transcatheter aortic valve replacement. JACC Cardiovasc Interv. 2019; 12(21): 2119-2129, doi: 10.1016/j.jcin.2019.06.019, indexed in Pubmed: 31629743.

2. Delgado V, Di Biase L, Leung M, et al. Structure and function of the left atrium and left atrial appendage: $\mathrm{AF}$ and stroke implications. J Am Coll Cardiol. 2017; 70(25): 3157-3172, doi: 10.1016/j. jacc.2017.10.063, indexed in Pubmed: 29268928.

3. Guo Yk, Yang $\mathrm{Zg}$, Shao H, et al. Right ventricular dysfunction and dilatation in patients with mitral regurgitation: analysis using ECG-gated multidetector row computed tomography. Int J Cardiol. 2013; 167(4): 1585-1590, doi: 10.1016/j.ijcard.2012.04.104, indexed in Pubmed: 22578735.

4. Li YG, Gong CQ, Zhao MZ, et al. Cardiac EP Study Group. Determinants of postoperative left atrial structural reverse remodeling in patients undergoing combined catheter ablation of atrial fibrillation and left atrial appendage closure procedure. J Cardiovasc Electrophysiol. 2019; 30(10): 1868-1876, doi: 10.1111/jce.14094, indexed in Pubmed: 31353748.

5. Kishima H, Mine T, Takahashi S, et al. Morphologic remodeling of left atrial appendage in patients with atrial fibrillation. Heart Rhythm. 2016; 13(9): 1823-1828, doi: 10.1016/j. hrthm.2016.06.009, indexed in Pubmed: 27291510.

6. Lisi M, Henein MY, Cameli M, et al. Severity of aortic stenosis predicts early post-operative normalization of left atrial size and function detected by myocardial strain. Int J Cardiol. 2013; 167(4): 1450-1455, doi: 10.1016/j.ijcard.2012.04.057, indexed in Pubmed: 22560912.

7. Megaly M, Garcia S, Anzia LE, et al. Detection of atrial fibrillation and atrial flutter by pacemaker device interrogation after transcatheter aortic valve replacement (TAVR): implications for management. J Invasive Cardiol. 2019; 31(7): E177-E183, indexed in Pubmed: 31257211.

8. Dahl JS, Videbæk L, Poulsen MK, et al. Noninvasive assessment of filling pressure and left atrial pressure overload in severe aortic valve stenosis: relation to ventricular remodeling and clinical outcome after aortic valve replacement. J Thorac Cardiovasc Surg. 2011; 142(3): e77-e83, doi: 10.1016/j.jtcvs.2011.01.032, indexed in Pubmed: 21353251.

9. Hatani T, Kitai T, Murai R, et al. Associations of residual left ventricular and left atrial remodeling with clinical outcomes in patients after aortic valve replacement for severe aortic stenosis. J Cardiol. 2016; 68(3): 241-247, doi: 10.1016/j.jjcc.2015.09.017, indexed in Pubmed: 26527112. 